\title{
Minireview
}

\section{Resistance to chemotherapy: new treatments and novel insights into an old problem}

\author{
S Raguz' and E Yagüe ${ }^{*, 2}$ \\ 'MRC Clinical Sciences Centre, Imperial College Faculty of Medicine, Hammersmith Hospital, Du Cane Road, London WI 2 ONN, UK; ${ }^{2}$ Division of \\ Surgery, Oncology, Reproductive Biology and Anaesthetics, Department of Oncology, Imperial College Faculty of Medicine, Hammersmith Hospital, \\ Du Cane Road, London WI 2 ONN, UK
}

\begin{abstract}
Resistance to cancer chemotherapeutic treatment is a common phenomenon, especially in progressive disease. The generation of cellular models of drug resistance has been pivotal in unravelling the main effectors of resistance to traditional chemotherapy at the molecular level (i.e. intracellular drug inactivation, detoxifying systems, defects in DNA repair, apoptosis evasion, membrane transporters and cell adhesion). The development of targeted therapies has also been followed by resistance, reminiscent of an evolutionary arms race, as exemplified by imatinib and other BCR-ABL inhibitors for the treatment of chronic myelogenous leukaemia. Although traditionally associated with the last stages of the disease, recent findings with minimally transformed pretumorigenic primary human cells indicate that the ability to generate drug resistance arises early during the tumorigenic process, before the full transformation. Novel technologies, such as genome profiling, have in certain cases predicted the outcome of chemotherapy and undoubtedly have tremendous potential for the future. In addition, the novel cancer stem cell paradigm raises the prospect of cell-targeted therapies instead of treatment directed against the whole tumour.
\end{abstract}

British Journal of Cancer (2008) 99, 387-39I. doi:I0.1038/sj.bjc.66045I0 www.bjcancer.com

Published online 29 July 2008

(c) 2008 Cancer Research UK

Keywords: drug resistance; chemotherapy response; cancer stem cells; genome profiling; chronic myelogenous leukaemia; breast cancer

Gilman and co-workers were the first to introduce chemotherapy into clinical practice at the end of the Second World War when they used nitrogen mustard to treat a patient with advanced malignant lymphoma. After an initial regression of the disease, a second course of therapy was given at a reduced dose due to the toxicity of the treatment, with an associated lesser therapeutic effect. By the time the third treatment was given, the tumour no longer responded to the chemotherapeutic agent (Goodman et al, 1946). Since then, chemotherapy has been one of the main therapeutic strategies in cancer treatment, but, to paraphrase Paul Ehrlich, resistance has followed as a faithful shadow.

Chemotherapeutic strategies have used a variety of drugs and hormonal agents that interfere with the basic machinery of the cell. Subsequent improved understanding of the molecular alterations present in the cancer cell has enabled the development of targeted therapies for some forms of cancer. Interestingly, resistance appears not only to traditional chemotherapy but also to targeted therapies such as tamoxifen, which targets the oestrogen receptor (ER) in breast cancer (Ali and Coombes, 2002); imatinib, which targets the kinase activity of the translocated $B C R-A B L$ in chronic myelogenous leukaemia (CML) (Weisberg et al, 2007); or gefitinib, which inhibits epidermal growth factor receptor (EGFR) kinase (Engelman et al, 2007).

*Correspondence: Dr E Yagüe; E-mail: ernesto.yague@imperial.ac.uk Received 30 November 2007; revised 16 April 2008; accepted 17 June 2008; published online 29 July 2008

\section{MECHANISMS OF RESISTANCE TO TRADITIONAL CHEMOTHERAPY}

Two main groups of factors contribute to the development of drug resistance. The first group includes pharmacological and physiological factors such as drug metabolism and excretion, inadequate access of the drug to the tumour, inadequate infusion rate and inadequate route of delivery. These are extremely important issues not only in clinical practice but also fundamental in drug development (Garattini, 2007). The second group includes cell- or tissue-specific factors. The cytochrome $P 450$ enzymes, a multigene family of constitutive and inducible haem-containing oxidative enzymes from the liver, play an important role in the metabolism of a diverse range of xenobiotics and are often overexpressed in a variety of solid tumours in which they can contribute to drug resistance. Drug analogues of DNA precursors such as 5-fluorouracil and cytosine arabinoside require metabolic activation, and resistance can arise from modification of these activation pathways. Altered topoisomerase I and II activity prevents drugs such as doxorubicin, etoposide and camptothecin from binding the topoisomerase-DNA complex, allowing the broken strands to be repaired. Many anticancer drugs, such as platinum compounds, alkylating agents and nitrosoureas, cause direct damage to the structural integrity of the DNA, and resistance to these compounds results from activation of DNA repair systems. Regulation of cell death by evasion of apoptosis, necrosis, mitotic catastrophe (Mansilla et al, 2006) or evasion of senescence (Dimri, 2005) contributes towards drug resistance. In addition, the differential expression of membrane proteins such as solute carriers, channels 
and ATP-binding cassette (ABC) transporters (Gottesman et al, 2002; Huang et al, 2004) have all been demonstrated to play an important role in drug resistance. Although these mechanisms have been clearly demonstrated in cell culture, their relevance with the clinical outcome of chemotherapy is less clear (Cimoli et al, 2004; Uggla et al, 2007) and has only been demonstrated for some of these mechanisms (Clarke et al, 2005).

\section{MOLECULAR MECHANISMS OF RESISTANCE TO TARGETED CHEMOTHERAPY}

\section{CML and imatinib}

Chronic myelogenous leukaemia was the first human cancer to be associated with a consistent chromosomal abnormality, the Philadelphia chromosome, a translocation that juxtaposes the $3^{\prime}$ sequence from the $A B L 1$ proto-oncogene on chromosome 9 with the $5^{\prime}$ sequence from the $B C R$ gene on chromosome 22 . The resultant chimaeric BCR-ABL protein is a constitutively active protein tyrosine kinase with an important role in the regulation of cell growth (Melo and Barnes, 2007). Traditional therapy for CML includes initial allogenic stem-cell transplantation and interferon$\alpha$, followed by second-line treatment with hydroxyurea or busulfan in non-responsive patients. Imatinib mesylate (formerly STI571; Gleevec, Novartis, Basel, Switzerland) is a potent and highly specific competitive inhibitor of the BCR-ABL tyrosine kinase. Initially, it had a high rate of cytogenetic and haematologic responses in patients with chronic-phase CML in whom previous therapy had failed, and its use has revolutionised the management and clinical expectations of CML patients. Unfortunately, not long after its initial use, resistance to imatinib was demonstrated in CML patients (Gorre et al, 2001). Approximately 50\% of imatinibresistant CML patients carry a resistance-associated point mutation in $B C R-A B L$, which interferes with imatinib binding. More than 50 different resistance-associated point mutations have been described to date. These findings have spurred the development of second-generation BCR-ABL inhibitors such as BMS-354825, which has a two-log increased potency relative to imatinib and retains its inhibitory activity against 14 of 15 imatinib-resistant BCR-ABL mutants tested (Shah et al, 2004). However, resistance to some of these second-generation inhibitors, such as nilotinib and dasatinib, has already been described. This indicates that the potential for new drug-resistant point mutations in BCR-ABL persists and justifies the continued development of more potent BCR-ABL inhibitors (Weisberg et al, 2007). In the remaining $50 \%$ of imatinib-resistant patients with no $B C R-A B L$ mutations, $B C R-A B L$ gene amplification or overexpression at the mRNA and protein levels has been detected in clinical samples (Hochhaus et al, 2002). In addition, chromosomal aberrations, reduced intracellular uptake of imatinib and the disease phase have all been implicated in imatinib resistance (Nimmanapalli and Bhalla, 2002).

\section{Breast cancer, tamoxifen, aromatase inhibitors and trastuzumab}

Breast cancer accounts for one in four of all female cancers, making it by far the most common cancer in women in the western world, where one in nine women will develop the disease at some stage in their lives. Breast cancer treatment involves surgical removal of the tumour, although this is ineffective if malignant cells have escaped from the site of the primary tumour. Discovery of the involvement of the ovarian hormone oestrogen and its mechanism of action (Dickson and Lippman, 1995) paved the way for the development of therapies for ER-positive patients that inhibit oestrogen action. These therapies include tamoxifen, which blocks the ER, and the oestrogen synthetase (aromatase) inhibitors formestane and exemestane, which inhibit oestrogen synthesis
(Ali and Coombes, 2002). Despite the huge improvement in cancer survival due to tamoxifen treatment, some patients relapse and the use of sequential therapy with exemestane after 2-3 years of tamoxifen treatment has improved disease-free survival, as compared with the standard 5 years of tamoxifen treatment alone (Coombes et al, 2004). The use of endocrine agents has markedly reduced the number of deaths from breast cancer over the past decades. However, in many cases, these therapies fail due to recurrent endocrine-resistant tumours, and much effort is being made to elucidate the mechanisms that underlie resistance to endocrine therapies (Weinberg et al, 2005). Altered growth factor signalling, notably EGFR (Schiff et al, 2005) and insulin-like growth factor I receptor (IGF-IR) make a significant contribution to the development of antioestrogen resistance, and these have been reviewed recently (Baselga, 2006).

Up to $25 \%$ of patients diagnosed with breast cancer have tumours that overexpress the EGFR-2 (HER2 or Erb B-2). HER2positive breast cancer is highly proliferative, difficult to treat and confers a poor prognosis. Trastuzumab is a monoclonal antibody targeted against the HER2 tyrosine kinase receptor. The majority of patients with metastatic breast cancer, who initially respond to trastuzumab, develop resistance within 1 year of treatment initiation, and in the adjuvant setting, $15 \%$ of patients still relapse despite trastuzumab-based therapy. Preclinical studies have indicated several molecular mechanisms that could contribute to the development of trastuzumab resistance. One major determinant to resistance is increased signalling via the phosphatidylinositol 3-kinase/Akt pathway. This results in the activation of multiple receptor pathways, including HER2-related receptors and non-HER receptors such as the IGF-IR, which appear to be involved in a cross talk with HER2 in resistant cells (Berns et al, 2007). Alternatively, the loss of function of the tumour suppressor PTEN, the negative regulator of Akt, results in an increase in Akt signalling that leads to decreased trastuzumab sensitivity. Decreased interaction between trastuzumab and its target receptor HER2, which is due to steric hindrance of HER2 by cell-surface proteins such as mucin-4 (MUC4), can block the inhibitory actions of trastuzumab. Novel therapies targeted against these aberrant molecular pathways offer hope that the effectiveness and duration of response to trastuzumab can be greatly improved (Baselga, 2006; Nahta et al, 2006). As only about one-third of breast cancer patients overexpressing HER2 respond to trastuzumab monotherapy, the identification of predictive biomarkers that can more accurately select responders or non-responders is vital, not only to improve its therapeutic index, but also to gain insight into the molecular pathways involved in trastuzumab resistance and to rationally design successful combination therapies.

\section{THE ABILITY TO ACQUIRE DRUG RESISTANCE ARISES EARLY DURING THE TUMORIGENESIS PROCESS}

The use of drug-resistant derivatives from human and other mammalian cell lines has been of paramount importance for the unravelling of many of the mechanisms of cancer drug resistance highlighted above. However, they have proved less successful in identifying the ultimate upstream regulators controlling these events, and as such, how drug resistance arises is still unresolved. Most cellular models of drug resistance have been developed from transformed cell lines isolated from patients at a late stage in cancer progression and whose tumours already exhibit a plethora of karyotypic and physiologic abnormalities. Recently, several groups have shown that it is possible to transform primary human cells into fully tumorigenic cells by altering a small number of defined pathways ex vivo. This cellular model of tumorigenesis (Figure 1A) was first described by Hahn et al (1999) in human BJ fibroblasts and embryonic kidney epithelial cells by expressing the 
A

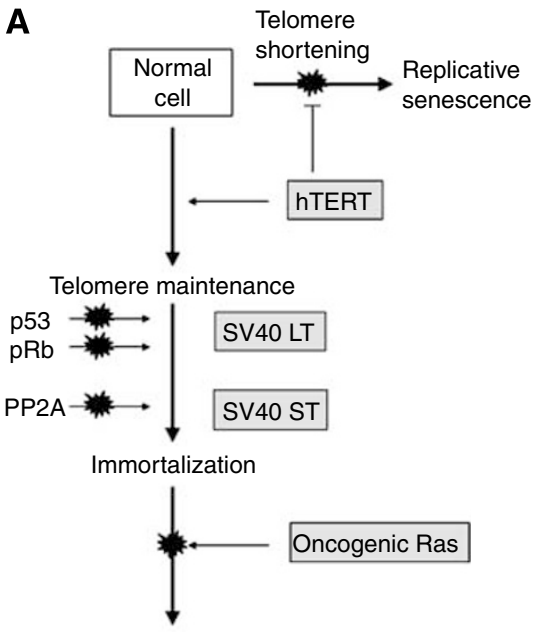

B

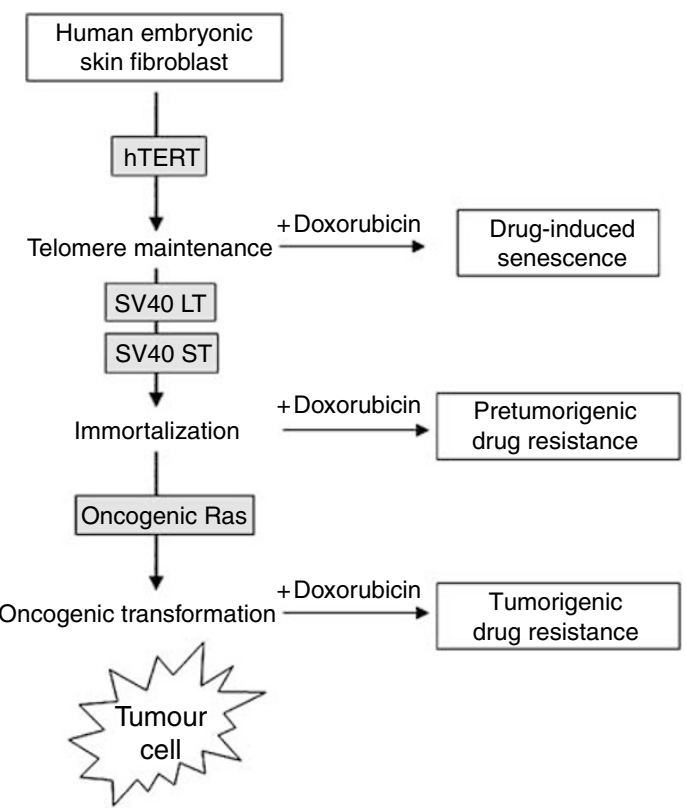

Figure I Cellular model of tumorigenesis and pretumorigenic drug resistance. (A) This model was first described by Hahn et al (1999) in human BJ fibroblasts and embryonic kidney epithelial cells. Normal cells are transformed by expressing the catalytic subunit of telomerase (to avoid replicative senescence), SV40 LT (which binds and inactivates the tumour suppressors p53 and pRb controlling the DNA repair and GI cell-cycle checkpoints, respectively) and ST (which binds and inactivates PP2A, a serine/threonine phosphatase involved in several signalling pathways) proteins and oncogenic ras. Since then, the model has been validated by transforming primary epithelial cells from breast, prostate, ovary and lung (Boehm and Hahn, 2005). (B) Complete tumorigenic transformation is not a prerequisite for the acquisition of drug resistance. When a series of pretumorigenic and minimally transformed tumorigenic cells derived from human embryonic skin fibroblasts are treated with doxorubicin, drug-resistant cells can be obtained from fully tumorigenic as well as pretumorigenic cells. Cells at the early stages of transformation, that is, those in which hTERT (telomerase) has been ectopically expressed, do not die due to the action of the drug, but become senescent (drug-induced sencescence). Disruption of the pathways controlled by the tumour suppressors $\mathrm{p} 53$ and $\mathrm{pRb}$ is necessary and sufficient to set the conditions for the acquisition of drug resistance. The diagram is based on data from Yagüe et al (2007).

catalytic subunit of telomerase (to avoid replicative senescence), SV40 large T-antigen (which binds and inactivates the tumour suppressors $\mathrm{p} 53$ and $\mathrm{pRb}$ controlling the DNA repair and G1 cell cycle checkpoints, respectively) and small t-antigen (which binds and inactivates PP2A, a serine/threonine phosphatase involved in several signalling pathways) and oncogenic ras. Primary human epithelial cells from the mammary gland, prostate, ovary, trachea and bronchia have now been transformed by introducing these or similar sets of genes (Boehm and Hahn, 2005). Although somatic p53 missense mutations are found in approximately $50 \%$ of human cancers, the p53 pathway can also be inactivated in wild-type p53carrying tumours by $\mathrm{p} 53$ destabilisation via indirect mechanisms such as $M D M 2 / M D M X$ amplification. In addition, most wild-type p53 types of cancer harbour alternative genetic alterations such as mutations in $A P C$ in colon cancer, BRCA1 and BRCA2 in breast cancer, and $B-R A F$ in melanoma. As the p53 network is closely linked to many other cellular pathways, it is likely that defects in any of these pathways could alter p53 function (Soussi and Wiman, 2007).

The development of these minimally transformed cells has, for the first time, allowed us to ask whether the capacity to develop drug resistance arises before or after tumorigenic transformation, and what is the minimum number of altered pathways required to permit this event. In a series of progressively transformed embryonic skin fibroblasts, it has been found that the minimum number of genetic transformations necessary for a primary cell to become drug resistant, in addition to hTERT expression, is inactivation of the pathways controlled by p53 and pRb (Yagüe et al, 2007), confirming the pivotal roles of p53 and pRb in deciding cell fate after drug treatment: senescence, apoptosis or drug resistance (Dimri, 2005). Thus, in this cell model, the ability to acquire drug resistance is not, as previously supposed, a late event in tumorigenesis resulting from gross genetic instability, but is intrinsic to the early steps in the tumorigenic pathway necessary for transformation and can arise earlier than the full malignant transformation (Figure 1B).

These findings with minimally transformed fibroblasts need to be confirmed and extended to other epithelial cancer cell models and to drugs with different modes of action to generalise their relevance. However, they have opened the possibility to analyse the ultimate controllers in the development of drug resistance.

\section{CANCER STEM CELLS AND DRUG RESISTANCE}

The cancer stem cell (CSC) hypothesis states that many, if not all, cancers contain a minority population of transformed self-renewing stem cells. These CSCs are responsible for sustaining the tumour as well as giving rise to proliferating but progressively differentiating cells constituting the tumour mass (Burkert et al, 2006; Li and Neaves, 2006). Cancer stem cells retain the essential property of self-protection through the activity of multiple drug resistance transporters such as ABCB1 (P-glycoprotein) and/or ABCG2 (Breast Cancer Resistance Protein-1, BCRP1). The latter is responsible for the side-population (SP) phenotype detected in both normal and acute myeologenous leukaemia (AML)

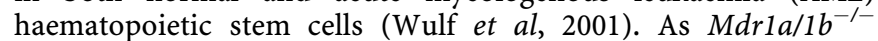
(P-glycoprotein-deficient) mice are able to display a normal SP phenotype that disappears when Abcg2 is knocked down (Zhou et al, 2002), expression of ABCG2 and Hoechst 33342 efflux are two of the best markers of these cells. To date, the existence of CSCs has been demonstrated in AML and CML, in brain and gastrointestinal tumours, and in lung and breast cancer (de Jonge-Peeters et al, 2007).

A connection between CSCs and drug resistance is thought to exist due to the expression of many of the membrane transporters. 
In fact, the whole drug resistance concept has been revised incorporating the CSC paradigm (Dean et al, 2005; Donnenberg and Donnenberg, 2005). According to the acquired resistance stem-cell model, CSCs, which express drug transporters, are present in the original tumour mass and survive chemotherapy, whereas the committed but variably differentiated cells are killed. These cells reform a heterogeneous drug resistant tumour composed of CSCs and a committed but variably differentiated offspring. In addition, mutation in the surviving CSCs can arise expanding the drug-resistant phenotype. However, the CSC hypothesis does not account for resistance that develops in certain cancers following chemotherapy in which all cancer cells (not just stem cells) become resistant. Such intrinsic drug resistance is in many cases, such as colon and liver cancer, due to the function of $A B C$ transporters, which are already highly expressed in the healthy tissues.

\section{DRUG RESISTANCE IN THE CLINIC AND ITS REVERSAL}

Cell culture systems and animal models have been pivotal in defining the main molecular and cellular mechanisms responsible for the drug-resistance phenotype. With them it has been relatively straight forward to demonstrate that a particular molecule (i.e. P-glycoprotein or p53) is the effector of drug resistance, due to the ease of performing knockout or ectopic expression experiments. However, the situation in the clinic is far more complicated, not only because of the lack of sensitivity and the absence of appropriate detection techniques from clinical samples, but also because the association of a particular drug-resistance effector does not necessarily correlate with an alteration in the chemotherapy response (Cimoli et al, 2004). Despite this, a negative correlation has been unequivocally demonstrated between P-glycoprotein expression and chemotherapy response in AML (Gottesman et al, 2002) and breast cancer (Clarke et al, 2005).

Since the discovery of P-glycoprotein in the early 1980s, most agents tested for the reversal of multidrug resistance in the clinic have aimed at inhibiting P-glycoprotein function. The first generation of P-glycoprotein inhibitors included verapamil, quinine and cyclosporine, which were already approved for other medical purposes. Although these compounds proved to be ineffective or toxic at the doses required to attenuate P-glycoprotein function, some clinical trials indicated that modulation of P-glycoprotein function could be achieved (Gottesman et al, 2002). This encouraged the development of a second-generation of modulators, such as the cyclosporine analogue PSC-833 (Valspodar), aimed at avoiding the toxic side effects seen in the first generation. However, the development of second-generation inhibitors has now been discontinued, mainly due to their limited success in clinical trials (PSC-833 induced pharmacological interactions that limited drug clearance and metabolism of the chemotherapeutic agent, thereby elevating plasma concentrations beyond acceptable toxicity). Third-generation inhibitors have been designed for low pharmacokinetic interaction, and inhibition of cytochrome P450 3A has been avoided with compounds such as laniquidar (R101933), oc144-093 (ONT-093), zosuquidar (LY335979), elacridar (GF-120918) and tariquidar (XR9576). A further generation of inhibitors acts on a broader range of $\mathrm{ABC}$ transporters. These include biricodar (VX-710) and GF-120918, which modulate not only P-glycoprotein but also MRP1 and ABCG2, respectively. Most clinical trial end points have not been analysed yet; for an extended discussion of P-glycoprotein inhibitors in the clinic see Szakacs et al (2006).

There are many different possible reasons for the failure of phase III clinical trial targeting P-glycoprotein. These include multifactorial mechanisms of resistance, toxicity of the inhibitors and unfavourable pharmacological interactions, as well as a poor clinical trial design.
The latter is exemplified by the phase III clinical trial using tariquidar as an adjunctive treatment in combination with first-line chemotherapy for patients with non-small cell lung carcinoma, in which there is no strong evidence to suggest that in this type of cancer, P-glycoprotein is expressed to a significant extent (Szakacs et al, 2006). Even AML patients, in which P-glycoprotein expression affects the outcome of chemotherapy, are not routinely phenotyped, and current efforts to develop simple, intercentre reproducible protocols to detect P-glycoprotein in AML blasts have been developed (Pallis et al, 2005). In such a way, trial organisers can have the choice of whether to give P-glycoprotein modulators to an unsorted cohort or to P-glycoprotein-positive patients only.

Other alternative approaches to target P-glycoprotein-mediated drug resistance could involve the development of agents to interfere with any one of the regulatory steps in P-glycoprotein expression: transcription, mRNA turnover, translation, protein processing and turnover. Of these, the only one under trial in soft tissue sarcoma (currently phase II) is ecteinascidin 743 , a natural product isolated from the marine organism Ecteinascidia turbinate. Ecteinascidin 743 interferes with the activation of $A B C B 1$ via the stress-responsive enhanceosome complex (Le Cesne et al, 2005).

Genome-wide expression profiling has been used to study drug resistance, and the foreseeable complexity of their mechanisms has been revealed. In addition to the corroboration of traditionally associated genes, these studies have given new insight into the regulatory networks controlling drug resistance (Turton et al, 2001). The most interesting application of the novel genomic technologies has been in the clinical arena, where molecular signatures have been used not only to characterise neoplastic transformation (Sotiriou et al, 2003) and resistant tumours (Jansen et al, 2005) but also, and most importantly, to predict the outcome of chemotherapy (Alaoui-Jamali et al, 2004). Whether these novel technologies will gain acceptance in the routine diagnosis of cancer will depend greatly on whether their current costs can be reduced (Sotiriou and Piccart, 2007).

\section{CONCLUSIONS AND PERSPECTIVES}

Many years have passed since the first description of cancer as an evolutionary process (Nowell, 1976). With the establishment of the CSC paradigm, new insights into the relative high frequency of cancer in humans and the pitfalls of many cancer treatments have been put forward based on Darwinian selection (Greaves, 2007). Although there are many differences between neoplastic and organismal evolution, the lack of cellular controls to maintain genomic stability, telomere length, repair of DNA damage or cell cycle regulation discussed above set up the conditions for genetic diversity as a source of clonal evolution. Treatment with chemotherapeutic agents promotes an evolutionary arms race exemplified by the resistance to imatinib and the generation of novel derivatives in CML. Studies using cell model systems have shown that chemotherapy resistance is intrinsic to the tumorigenesis process and can even arise before malignant transformation. Thus, possible solutions lie in the development of novel approaches, based mainly on immunological and gene therapy techniques, aiming to at least partially restore some of the normal cellular controls, although cancer gene therapy is still in its infancy. Whether these or other still-to-come therapies will allow us to say in future years, paraphrasing the Borg from Star Trek, 'Resistance is futile' is difficult to foresee, but as Captain Jean-Luc Picard stated 'Things are only impossible until they're not'.

\section{ACKNOWLEDGEMENTS}

This work is funded by the UK Medical Research Council. We thank three anonymous reviewers, $\mathrm{H}$ Unsworth and EJ Andress for their comments to improve this minireview. 


\section{REFERENCES}

Alaoui-Jamali MA, Dupre I, Qiang H (2004) Prediction of drug sensitivity and drug resistance in cancer by transcriptional and proteomic profiling. Drug Resist Updat 7: 245-255

Ali S, Coombes RC (2002) Endocrine-responsive breast cancer and strategies for combating resistance. Nat Rev Cancer 2: 101-112

Baselga J (2006) Targeting tyrosine kinases in cancer: the second wave. Science 312: $1175-1178$

Berns K, Horlings HM, Hennessy BT, Madiredjo M, Hijmans EM, Beelen K, Linn SC, Gonzalez-Angulo AM, Stemke-Hale K, Hauptmann M, Beijersbergen RL, Mills GB, van de Vijver MJ, Bernards R (2007) A functional genetic approach identifies the PI3K pathway as a major determinant of trastuzumab resistance in breast cancer. Cancer Cell 12: $395-402$

Boehm JS, Hahn WC (2005) Understanding transformation: progress and gaps. Curr Opin Genet Dev 15: $13-17$

Burkert J, Wright NA, Alison MR (2006) Stem cells and cancer: an intimate relationship. J Pathol 209: 287-297

Cimoli G, Malacarne D, Ponassi R, Valenti M, Alberti S, Parodi S (2004) Meta-analysis of the role of p53 status in isogenic systems tested for sensitivity to cytotoxic antineoplastic drugs. Biochim Biophys Acta 1705: $103-120$

Clarke R, Leonessa F, Trock B (2005) Multidrug resistance/P-glycoprotein and breast cancer: review and meta-analysis. Semin Oncol 32: S9-S15

Coombes RC, Hall E, Gibson LJ, Paridaens R, Jassem J, Delozier T, Jones SE, Alvarez I, Bertelli G, Ortmann O, Coates AS, Bajetta E, Dodwell D, Coleman RE, Fallowfield LJ, Mickiewicz E, Andersen J, Lonning PE, Cocconi G, Stewart A, Stuart N, Snowdon CF, Carpentieri M, Massimini G, Bliss JM, van de Velde C (2004) A randomized trial of exemestane after two to three years of tamoxifen therapy in postmenopausal women with primary breast cancer. N Engl J Med 350: 1081-1092

de Jonge-Peeters SDPWM, Kuipers F, de Vries EGE, Vellenga E (2007) ABC transporter expression in hematopoietic stem cells and the role in AML drug resistance. Crit Rev Oncol Hematol 62: 214-226

Dean M, Fojo T, Bates S (2005) Tumour stem cells and drug resistance. Nat Rev Cancer 5: 275-284

Dickson RB, Lippman ME (1995) Growth factors in breast cancer. Endocr Rev 16: $559-589$

Dimri GP (2005) What has senescence got to do with cancer? Cancer Cell 7: $505-512$

Donnenberg VS, Donnenberg AD (2005) Multiple drug resistance in cancer revisited: the cancer stem cell hypothesis. J Clin Pharmacol 45: 872-877

Engelman JA, Zejnullahu K, Mitsudomi T, Song Y, Hyland C, Park JO, Lindeman N, Gale CM, Zhao X, Christensen J, Kosaka T, Holmes AJ, Rogers AM, Cappuzzo F, Mok T, Lee C, Johnson BE, Cantley LC, Janne PA (2007) MET amplification leads to gefitinib resistance in lung cancer by activating ERBB3 signaling. Science 316: 1039-1043

Garattini S (2007) Pharmacokinetics in cancer chemotherapy. Eur J Cancer 43: $271-282$

Goodman LS, Wintrobe MM, Dameshek W, Goodman MJ, Gilman A, McLennan MT (1946) Nitrogen mustard therapy. JAMA 132: $126-132$

Gorre ME, Mohammed M, Ellwood K, Hsu N, Paquette R, Rao PN, Sawyers CL (2001) Clinical resistance to STI-571 cancer therapy caused by BCR-ABL gene mutation or amplification. Science 293: 876-880

Gottesman MM, Fojo T, Bates SE (2002) Multidrug resistance in cancer: role of ATP-dependent transporters. Nat Rev Cancer 2: 48-58

Greaves M (2007) Darwinian medicine: a case for cancer. Nat Rev Cancer 7: $213-221$

Hahn WC, Counter CM, Lundberg AS, Beijersbergen RL, Brooks MW, Weinberg RA (1999) Creation of human tumour cells with defined genetic elements. Nature 400: 464-468

Hochhaus A, Kreil S, Corbin AS, La Rosee P, Muller MC, Lahaye T, Hanfstein B, Schoch C, Cross NC, Berger U, Gschaidmeier H, Druker BJ, Hehlmann R (2002) Molecular and chromosomal mechanisms of resistance to imatinib (STI571) therapy. Leukemia 16: 2190-2196

Huang Y, Anderle P, Bussey KJ, Barbacioru C, Shankavaram U, Dai Z, Reinhold WC, Papp A, Weinstein JN, Sadee W (2004) Membrane transporters and channels: role of the transportome in cancer chemosensitivity and chemoresistance. Cancer Res 64: 4294-4301

Jansen MP, Foekens JA, van Staveren IL, Dirkzwager-Kiel MM, Ritstier K, Look MP, Meijer-van Gelder ME, Sieuwerts AM, Portengen H, Dorssers
LC, Klijn JG, Berns EM (2005) Molecular classification of tamoxifenresistant breast carcinomas by gene expression profiling. J Clin Oncol 23: $732-740$

Le Cesne A, Blay JY, Judson I, Van Oosterom A, Verweij J, Radford J, Lorigan P, Rodenhuis S, Ray-Coquard I, Bonvalot S, Collin F, Jimeno J, Di Paola E, Van Glabbeke M, Nielsen OS (2005) Phase II study of ET-743 in advanced soft tissue sarcomas: a European Organisation for the Research and Treatment of Cancer (EORTC) soft tissue and bone sarcoma group trial. J Clin Oncol 23: $576-584$

Li L, Neaves WB (2006) Normal stem cells and cancer stem cells: the niche matters. Cancer Res 66: 4553-4557

Mansilla S, Bataller M, Portugal J (2006) Mitotic catastrophe as a consequence of chemotherapy. Anticancer Agents Med Chem 6: 589-602

Melo JV, Barnes DJ (2007) Chronic myeloid leukaemia as a model of disease evolution in human cancer. Nat Rev Cancer 7: 441-453

Nahta R, Yu D, Hung MC, Hortobagyi GN, Esteva FJ (2006) Mechanisms of disease: understanding resistance to HER2-targeted therapy in human breast cancer. Nat Clin Pract Oncol 3: 269-280

Nimmanapalli R, Bhalla K (2002) Mechanisms of resistance to imatinib mesylate in Bcr-Abl-positive leukemias. Curr Opin Oncol 14: 616-620

Nowell PC (1976) The clonal evolution of tumor cell populations. Science 194: $23-28$

Pallis M, Fisher J, Truran L, Grundy M, Russell N, Burnett A (2005) Reproducible measurements of AML blast p-glycoprotein function in 2 center analyses. Blood 105: $1367-1368$

Schiff R, Massarweh SA, Shou J, Bharwani L, Arpino G, Rimawi M, Osborne CK (2005) Advanced concepts in estrogen receptor biology and breast cancer endocrine resistance: implicated role of growth factor signaling and estrogen receptor coregulators. Cancer Chemother Pharmacol 56(Suppl 1): $10-20$

Shah NP, Tran C, Lee FY, Chen P, Norris D, Sawyers CL (2004) Overriding imatinib resistance with a novel ABL kinase inhibitor. Science 305: $399-401$

Sotiriou C, Neo SY, McShane LM, Korn EL, Long PM, Jazaeri A, Martiat P, Fox SB, Harris AL, Liu ET (2003) Breast cancer classification and prognosis based on gene expression profiles from a population-based study. Proc Natl Acad Sci USA 100: 10393-10398

Sotiriou C, Piccart MJ (2007) Taking gene-expression profiling to the clinic: when will molecular signatures become relevant to patient care? Nat Rev Cancer 7: $545-553$

Soussi T, Wiman KG (2007) Shaping genetic alterations in human cancer: the p53 mutation paradigm. Cancer Cell 12: 303-312

Szakacs G, Paterson JK, Ludwig JA, Booth-Genthe C, Gottesman MM (2006) Targeting multidrug resistance in cancer. Nat Rev Drug Discov 5: $219-234$

Turton NJ, Judah DJ, Riley J, Davies R, Lipson D, Styles JA, Smith AG, Gant TW (2001) Gene expression and amplification in breast carcinoma cells with intrinsic and acquired doxorubicin resistance. Oncogene 20: $1300-1306$

Uggla B, Tina E, Nahi H, Paul C, Hoglund M, Sirsjo A, Tidefelt U (2007) Topoisomerase IIalpha mRNA and protein expression vs in vitro drug resistance and clinical outcome in acute leukaemia. Int J Oncol 31: $153-160$

Weinberg OK, Marquez-Garban DC, Pietras RJ (2005) New approaches to reverse resistance to hormonal therapy in human breast cancer. Drug Resist Updat 8: 219-233

Weisberg E, Manley PW, Cowan-Jacob SW, Hochhaus A, Griffin JD (2007) Second generation inhibitors of BCR-ABL for the treatment of imatinib-resistant chronic myeloid leukaemia. Nat Rev Cancer 7: $345-356$

Wulf GG, Wang RY, Kuehnle I, Weidner D, Marini F, Brenner MK, Andreeff M, Goodell MA (2001) A leukemic stem cell with intrinsic drug efflux capacity in acute myeloid leukemia. Blood 98: 1166-1173

Yagüe E, Arance A, Kubitza L, O'Hare M, Jat P, Ogilvie CM, Hart IR, Higgins CF, Raguz S (2007) Ability to acquire drug resistance arises early during the tumorigenesis process. Cancer Res 67: 1130-1137

Zhou S, Morris JJ, Barnes Y, Lan L, Schuetz JD, Sorrentino BP (2002) Bcrp1 gene expression is required for normal numbers of side population stem cells in mice, and confers relative protection to mitoxantrone in hematopoietic cells in vivo. Proc Natl Acad Sci USA 99: 12339-12344 